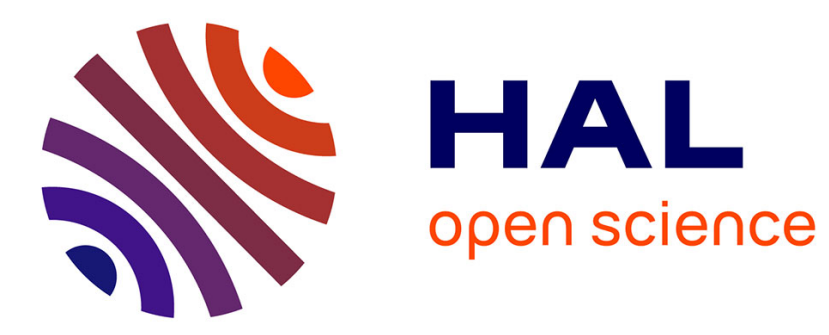

\title{
Stress-induced magnetic textures and fluctuating chiral phase in MnGe chiral magnet
}

\author{
M Deutsch, Pierre Bonville, A. V. Tsvyashchenko, L. N. Fomicheva, F. \\ Porcher, F. Damay, S. Petit, Isabelle Mirebeau
}

\section{To cite this version:}

M Deutsch, Pierre Bonville, A. V. Tsvyashchenko, L. N. Fomicheva, F. Porcher, et al.. Stress-induced magnetic textures and fluctuating chiral phase in MnGe chiral magnet. Physical Review B: Condensed Matter and Materials Physics (1998-2015), 2014, 90, pp.144401. 10.1103/PhysRevB.90.144401 . cea01377039

\section{HAL Id: cea-01377039 https://hal-cea.archives-ouvertes.fr/cea-01377039}

Submitted on 6 Oct 2016

HAL is a multi-disciplinary open access archive for the deposit and dissemination of scientific research documents, whether they are published or not. The documents may come from teaching and research institutions in France or abroad, or from public or private research centers.
L'archive ouverte pluridisciplinaire HAL, est destinée au dépôt et à la diffusion de documents scientifiques de niveau recherche, publiés ou non, émanant des établissements d'enseignement et de recherche français ou étrangers, des laboratoires publics ou privés. 


\title{
Stress-induced magnetic textures and fluctuating chiral phase in MnGe chiral magnet
}

\author{
M. Deutsch, ${ }^{1}$ P. Bonville, ${ }^{2}$ A. V. Tsvyashchenko, ${ }^{3,4}$ L. N. Fomicheva, ${ }^{3}$ F. Porcher, ${ }^{1}$ F. Damay, ${ }^{1}$ S. Petit, ${ }^{1}$ and I. Mirebeau ${ }^{1, *}$ \\ ${ }^{1}$ CEA, Centre de Saclay, DSM/IRAMIS/ Laboratoire Léon Brillouin, 91191 Gif-sur-Yvette, France \\ ${ }^{2}$ CEA, Centre de Saclay, DSM/IRAMIS/SPEC, 91191 Gif-sur-Yvette, France \\ ${ }^{3}$ Vereshchagin Institute for High Pressure Physics, Russian Academy of Sciences, 142190, Troitsk, Moscow, Russia \\ ${ }^{4}$ Skobeltsyn Institute of Nuclear Physics, MSU, Vorob'evy Gory 1/2, 119991 Moscow, Russia
}

(Received 23 June 2014; published 1 October 2014)

\begin{abstract}
We have studied the MnGe chiral magnet below $T_{\mathrm{N}}=170 \mathrm{~K}$, by magnetic measurements, Mössbauer spectroscopy, and by neutron diffraction at ambient and under nonhydrostatic pressure. At ambient pressure, we observe the coexistence of two magnetic phases belonging to the same crystal phase in a large temperature range (down to $100 \mathrm{~K}$ ) below $T_{\mathrm{N}}$ : ferromagnetically correlated rapidly fluctuating spins coexist with frozen spins involved in the helical order. Applying a uniaxial pressure component induces a strong magnetic texture, where most of the helical axes reorient along the stress axis. The magnetic texture persists in the fluctuating chiral state up to $T_{\mathrm{N}}$. Our results suggest that the zero field ground state at ambient pressure is a multidomain state consisting of helical domains with random orientations rather than a three-dimensional skyrmion lattice. They show the presence of an unusually broad transition to paramagnetism with a dynamical phase separation triggered by temperature.
\end{abstract}

DOI: 10.1103/PhysRevB.90.144401

PACS number(s): 75.30.Kz, 62.50.-p, 75.25.-j, 76.80.+y

\section{INTRODUCTION}

Chiral itinerant magnets of the B20 family now attract increasing attention due to their complex and highly tunable spin structures. Long wavelength helical modulations of the local magnetic moment result from the competition between ferromagnetism and weaker order anisotropy terms, since the lack of inversion symmetry of the cubic $P 22_{1} 3$ space group leads to nonvanishing chiral interactions. In some cases, peculiar spin textures called Skyrmions (SK) are stabilized, behaving as topologically protected objects much larger than the magnetic unit cell. Skyrmions, studied both in thin films and in the bulk, are now considered as possible building blocks for future spin-based electronics [1,2]. In thin films, where surface anisotropy plays the main role, SK were observed by electron microscopy $[3,4]$ and manipulated with ultra low current densities, yielding spin transfer torque and topological Hall effect (THE) [5,6], with promising applications to spintronics $[2,7]$.

In the bulk, helical structures are induced by the anisotropy of the spin-relativistic Dzyaloshinskii-Moriya (DM) interaction. The smaller crystalline field anisotropy pins the propagation vector of the helices along the crystal axes. In bulk $\mathrm{MnSi}$ and $\mathrm{FeGe}$, a triangular skyrmion lattice was revealed by single crystal neutron diffraction in applied field $[6,8,9]$. It was analyzed as a long range ordered multi- $k$ structure built from in-phase helices propagating along different directions [10]. This so-called A phase is induced by a magnetic field and is limited to a narrow temperature range just below the Néel temperature $T_{\mathrm{N}}$, being favored by thermal fluctuations. In $\mathrm{MnSi}$, the A phase extends under pressure as $T_{\mathrm{N}}$ decreases, approaching a quantum phase transition (QPT) reached at a critical pressure $\mathrm{P}_{c}$ of $1.4 \mathrm{GPa}$. Chiral fluctuations of isotropic character recalling skyrmions were reported either in the

\footnotetext{
*isabelle.mirebeau@cea.fr
}

non-Fermi liquid phase above $\mathrm{P}_{c}[11]$ or in the paramagnetic phase $[12,13]$.

Within the B20 family, MnGe stands apart due to its peculiar properties. Cubic MnGe exists in metastable and powdered state only, and must be synthesized under high temperature and high pressure conditions. It shows a strong exchange interaction, revealed by a high transition temperature $T_{\mathrm{N}}$ of about $170 \mathrm{~K}$ (instead of $29.5 \mathrm{~K}$ in $\mathrm{MnSi}$ ), and a large ordered Mn moment of about $1.8 \mu_{\mathrm{B}}$ at $2 \mathrm{~K}$ (compared to $0.4 \mu_{\mathrm{B}}$ at $2 \mathrm{~K}$ in $\mathrm{MnSi}$ ). The strong spin orbit coupling results in the shortest helix pitch of the series of B20 metals ( $29 \AA$ instead of $180 \AA$ in $\mathrm{MnSi}$ and $700 \AA$ An $\mathrm{FeGe}$ ) $[14,15]$. MnGe also exhibits a giant THE [14] and a topological Nernst effect [16], which makes it very promising for spintronic applications. Very recently, by applying high hydrostatic pressures, we observed the onset of a pressure induced collapse of the magnetic order in MnGe [17]. This collapse occurs in two steps, and may be associated with the specific band structure of MnGe, opening the possibility of a high spin-low spin transition $[17,18]$.

In MnGe, a skyrmion lattice was postulated to exist in the bulk from small angle neutron scattering (SANS) and resistivity measurements, and the measured giant THE was tentatively related to a high skyrmion density [14,19]. By contrast with the two-dimensional (2D) triangular SK lattice observed in $\mathrm{MnSi}$ and in all other B20 magnets, the proposed SK lattice in $\mathrm{MnGe}$ is 3D and cubic. Moreover, as a striking difference with other B20 magnets, it would extend in the whole phase diagram below $T_{\mathrm{N}}$ and down to $T=0$, even without magnetic field. In zero field, alternating skyrmions and antiskyrmions have been proposed, realizing a complex texture with no net magnetization [7]. However, in the absence of visualization by single crystal neutron diffraction, the existence of a SK lattice in bulk MnGe remains hypothetical.

In this paper, we first investigate the transition region in MnGe by combining magnetic susceptibility, magnetization, neutron scattering, and ${ }^{57} \mathrm{Fe}$ Mössbauer spectroscopy in a $\mathrm{Mn}_{0.98}{ }^{57} \mathrm{Fe}_{0.02} \mathrm{Ge}$ sample. We observe the coexistence of two 
types of magnetic phases below $T_{\mathrm{N}}$, in a large temperature range down to about $100 \mathrm{~K}$, namely rapidly fluctuating $\mathrm{Mn}$ moments and long range ordered "frozen" Mn moments. We determine the thermal evolution of the relative fraction and spin correlations of both phases. The analysis of the Mössbauer spectra also suggests the onset of a distortion at the Néel transition, in agreement with previous neutron diffraction data [15].

We also studied the influence of a uniaxial pressure component on the magnetic order, as directly probed by neutron diffraction. We show that a small uniaxial pressure is sufficient to induce a strong magnetic texture, clearly observed on a $2 \mathrm{D}$ multidetector, which persists up to $T_{\mathrm{N}}$. The anisotropy of the intensity distribution is readily interpreted by a reorientation of the helical axes along the axis of the pressure cell, and numerical integrations show that the great majority of the helices reorient under stress. Our results strongly suggest that the zero field ground state at ambient pressure is a multidomain state, consisting of helical domains with random orientations, rather than a 3D SK lattice.

Altogether, our results support the existence in $\mathrm{MnGe}$ of a fluctuating chiral state, extending over a large temperature range from about $100 \mathrm{~K}$ up to well above $T_{\mathrm{N}}$. Below $T_{\mathrm{N}}$, this state is inhomogeneous, with coexistence of fluctuating and frozen magnetic phases, possibly favored by magnetoelastic interactions. We discuss its possible nature in the scope of the available data on $\mathrm{MnGe}$.

\section{SAMPLE SYNTHESIS AND MAGNETIC MEASUREMENTS}

Polycrystalline MnGe was synthesized under $8 \mathrm{GPa}$ in a toroidal high-pressure apparatus by melting reaction with $\mathrm{Mn}$ and Ge. The purity of the constituents was $99.9 \%$ and $99.999 \%$ for $\mathrm{Mn}$ and $\mathrm{Ge}$, respectively. The pellets of well-mixed powdered constituents were placed in rocksalt pipe ampoules and then directly electrically heated to $T \simeq 1600{ }^{\circ} \mathrm{C}$. Then the samples were quenched to room temperature before releasing the applied pressure [20]. Two samples (called 1 and 2) doped with $2 \%$ at. ${ }^{57} \mathrm{Fe}$ were also synthesized for the Mössbauer experiments. The amount of impurity phases in these samples is estimated from $\mathrm{x}$ ray diffraction to less than $2 \%$.

The magnetic susceptibility was measured versus temperature in a field of $0.01 \mathrm{~T}$ with zero field cooling (ZFC) procedure. In all samples, the susceptibility curves (Fig. 1, upper panel) show a broad and high maximum at the Néel transition, namely $T_{\mathrm{N}}=170(5) \mathrm{K}$ for the neutron sample and the Mössbauer sample 1 , and $T_{\mathrm{N}}=150(5) \mathrm{K}$ for the Mössbauer sample 2 .

A second anomaly is observed at lower temperature near $T_{c} \simeq 40-50 \mathrm{~K}$, which is strongly enhanced in the sample 1 . The difference between samples arises from an unknown parameter in the synthesis, likely linked to the high-temperature high-pressure quench. Comparison between the susceptibility curves in samples 1 and 2 shows that their differences are not related to the $\mathrm{Fe}$ doping. These curves also differ in two pure MnGe samples [21]. As for the anomaly at $T_{c}$, it is strongly enhanced under pressure for a given sample [17]. This suggests relating the amplitude of this second anomaly to the mobility of the helical domain walls, and to the pinning by defects induced by thermal quench, pressure, or stress. The
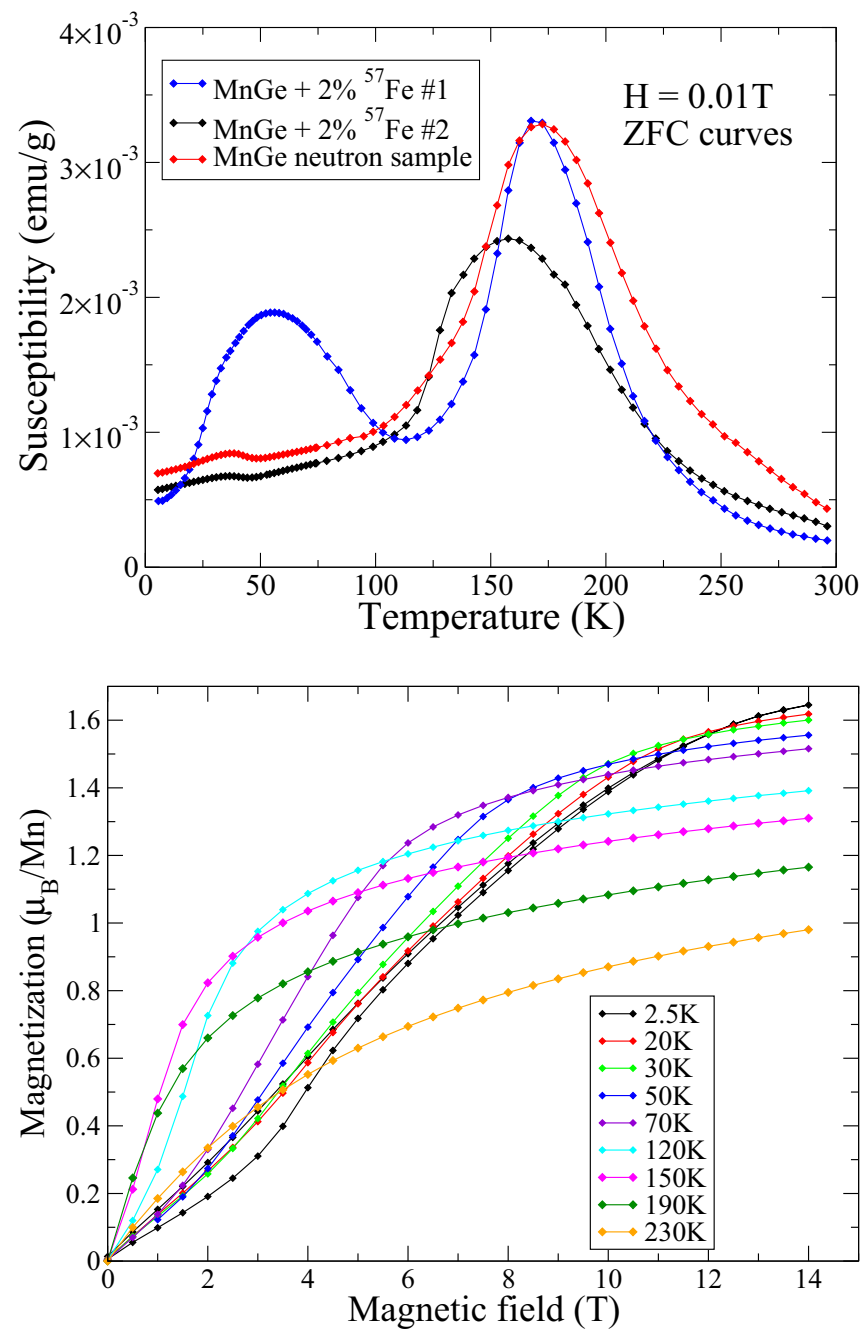

FIG. 1. (Color online) Upper panel: magnetic susceptibility curves in the pure MnGe neutron sample and in two samples doped with $2 \%$ at. ${ }^{57} \mathrm{Fe}$, with $\mathrm{ZFC}$ procedure and with a field of $0.01 \mathrm{~T}$. Lower panel: isothermal magnetization curves in the neutron $\mathrm{MnGe}$ sample at different temperatures.

temperature of this anomaly roughly coincides with the lock-in of the helical modulation, the neutron and Mössbauer results not being affected. The unusual width of the Néel transition, namely $T_{N} \pm 70 \mathrm{~K}$, is observed in all samples, showing that it is a genuine feature of MnGe. This unusually broad transition region is identified as the range of existence of the fluctuating moment phase as shown below.

Isothermal magnetization curves $m(H)$ were measured up to $14 \mathrm{~T}$. The lower panel of Fig. 1 shows the curves in the neutron MnGe sample (the magnetization in the Fe-doped sample shows the same features). Our data are in good agreement with the measurements reported in Ref. [14]. These curves can be divided into two sets: (i) a low temperature set below $70 \mathrm{~K}$, where the $m(H)$ curves increase slowly with the applied field, then show an inflection point near 2-3 T, and finally present an approach to saturation above a field of about $12 \mathrm{~T}$ at the lowest temperature; (ii) a high temperature set above $120 \mathrm{~K}$ where the $m(H)$ curves are quite different, increasing steadily with the field without any 


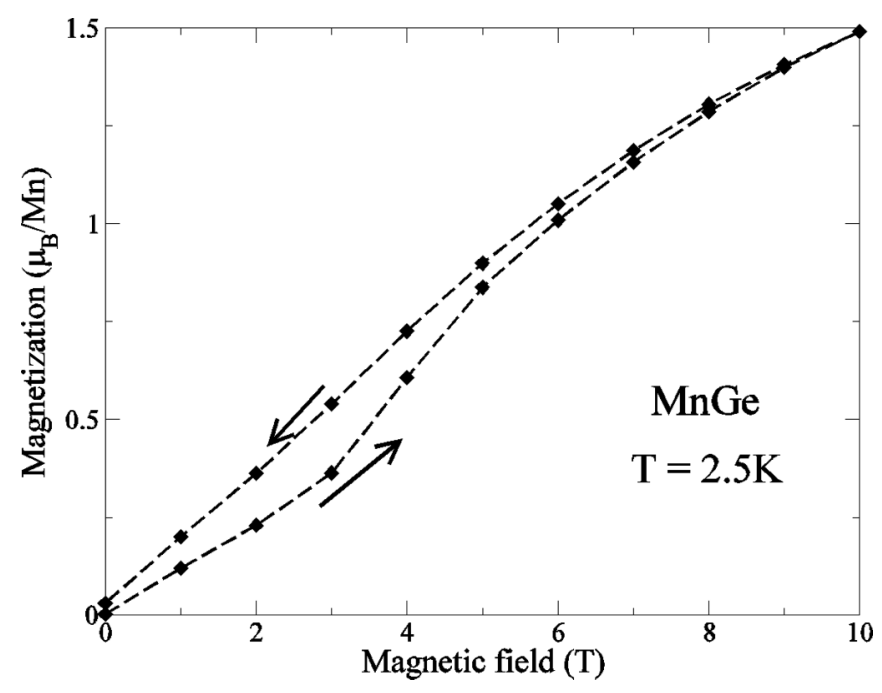

FIG. 2. Hysteretic behavior of MnGe magnetization at $2.5 \mathrm{~K}$.

inflection point and presenting a Brillouin-like shape. The temperature at which occurs this remarkable change of shape of the magnetization curves coincides with the onset of the rapidly fluctuating state as seen by Mössbauer spectroscopy and neutron diffraction (see below). At the highest field and lowest temperature, the system is close to a polarized ferromagnetic state with a moment value $\left(1.65 \mu_{\mathrm{B}} / \mathrm{Mn}\right)$ close to the spontaneous saturated moment of $1.80 \mu_{\mathrm{B}} / \mathrm{Mn}$ measured by neutron diffraction (see below). The saturation field and saturated magnetic moments are much higher in $\mathrm{MnGe}$ than in $\mathrm{MnSi}\left(0.5 \mathrm{~T}\right.$ and $0.4 \mu_{\mathrm{B}} / \mathrm{Mn}$, respectively).

The magnetization curves of the polycrystalline $\mathrm{MnGe}$ sample at low temperature show a hysteretic behavior (see Fig. 2): the first ZFC magnetization curve performed at $2.5 \mathrm{~K}$ presents a dip when increasing the field, which disappears when the field is decreased. This is considered as a signature of the helicoidal magnetic structure [14] with a significant in-plane anisotropy: as long as the field is sufficiently low, the Mn moments tend to remain close to the (001) planes resulting in a reduced susceptibility. On further increase of the field, the moments reorient towards the field direction adopting a conical structure ending in a ferromagnetically induced state, as we have seen, above about $12 \mathrm{~T}$. When the field is decreased, the dip is not observed since the moments recover smoothly their original positions. The irreversibility field for which the upward and downward magnetization curves coincide (about $7 \mathrm{~T}$ ) is quite high, pointing to a very strong planar anisotropy as compared to that in $\mathrm{MnSi}$, showing an irreversibility field of $0.1 \mathrm{~T}[22,23]$. The hysteresis has almost disappeared at $20 \mathrm{~K}$.

\section{MÖSSBAUER EXPERIMENTS}

The Mössbauer spectra were recorded in zero applied field in the temperature range from $4.2 \mathrm{~K}$ to $170 \mathrm{~K}$ in samples 1 and 2 . The spectra show qualitatively similar features in both samples. Since $T_{\mathrm{N}}$ in sample 1 is identical to $T_{\mathrm{N}}$ in the neutron sample, we show and discuss in the following only the spectra in sample 1 , for comparison with the neutron data.

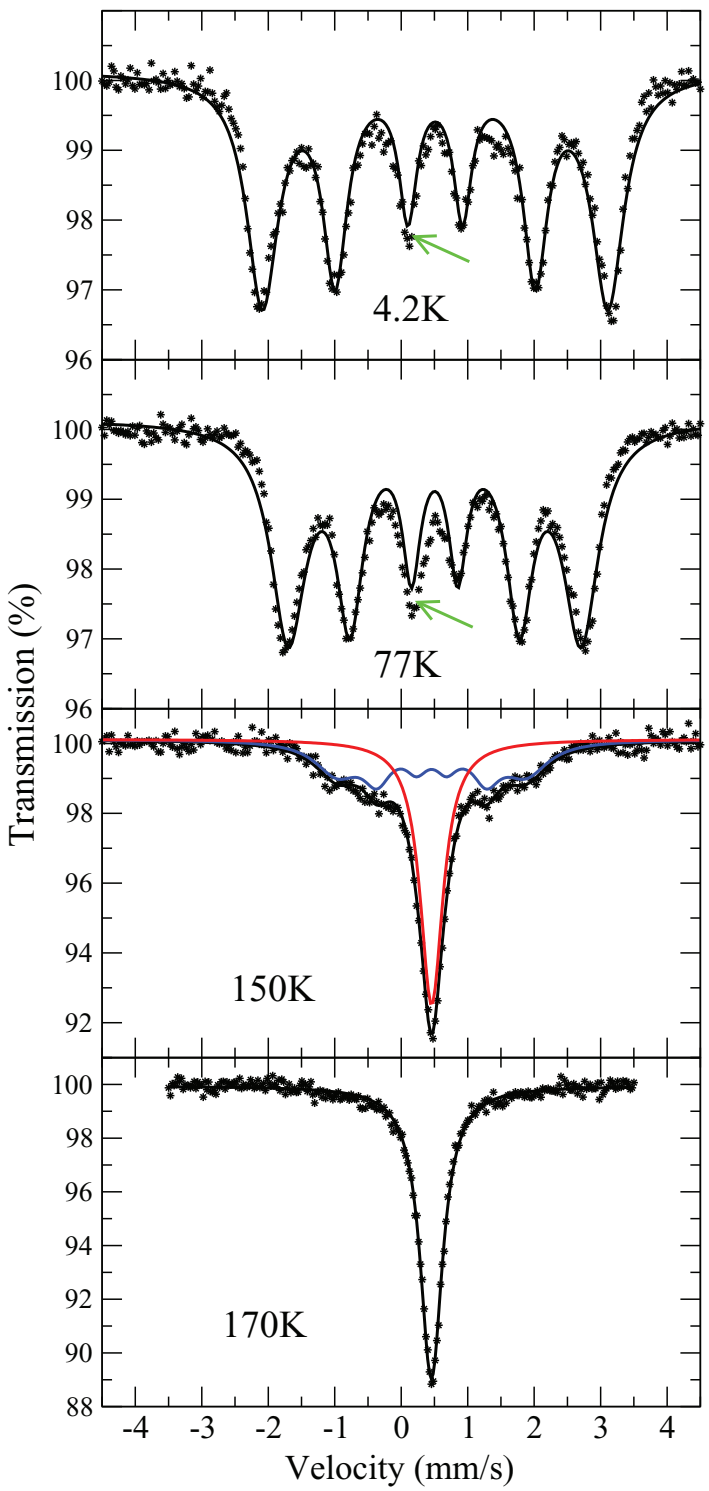

FIG. 3. (Color online) ${ }^{57} \mathrm{Fe}$ Mössbauer absorption spectra in ${ }^{57} \mathrm{Fe}$-doped $\mathrm{MnGe}$ sample 1 at selected temperatures. At 4.2 and $77 \mathrm{~K}$, the lines are fits to a standard six-line magnetic hyperfine pattern. At $150 \mathrm{~K}$, the fit corresponds to a superposition of a single narrow line (in red) and of a six-line hyperfine pattern (in blue). At $170 \mathrm{~K}$, the spectrum consists of a single narrow line.

Below $100 \mathrm{~K}$, the spectra are six-line slightly broadened magnetic hyperfine patterns. Above $110 \mathrm{~K}$, a single line appears in the spectra, with the same isomer shift as the magnetic spectrum, i.e., pertaining to the same crystallographic phase. The relative weight of this single line grows with increasing temperature at the expense of that of the magnetic pattern. At $170 \mathrm{~K}$, the single line alone is present (see Fig. 3). This single line corresponds to $\mathrm{Fe}$ nuclei which are either in a paramagnetic phase or which are submitted to a hyperfine field fluctuating rapidly with respect to the hyperfine Larmor time for ${ }^{57} \mathrm{Fe}: 10^{-8}$ s. In Fig. 4 are represented the thermal variations of the hyperfine field (left scale) and of the fluctuating fraction (right scale). The presence of the narrow single line alone at $170 \mathrm{~K}$ means that, at this temperature, the whole sample 


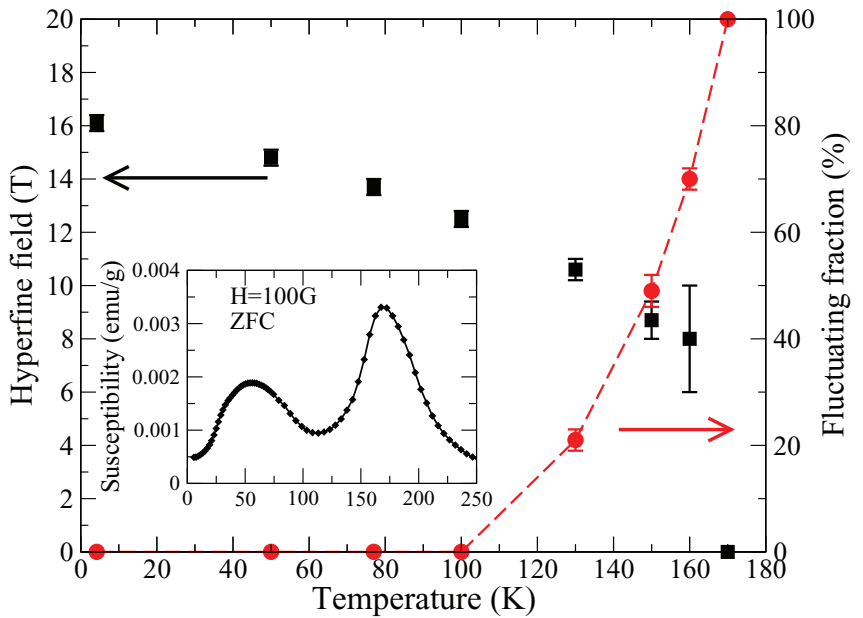

FIG. 4. (Color online) Thermal variations of the hyperfine field (black solid squares) and of the fluctuating fraction (red solid circles) deduced from the Mössbauer spectra in Fe-doped MnGe sample 1. The red dashed line is a guide for the eye. The inset shows the susceptibility vs temperature in Fe-doped MnGe sample 1.

is paramagnetic and that the electric field gradient (EFG) is actually zero in the paramagnetic phase. Comparing the thermal variation of the fluctuating fraction with that of the ZFC susceptibility (inset in Fig. 4), it is clear that the appearance of the phase with rapidly fluctuating moments around $110 \mathrm{~K}$ corresponds to the onset of the unusually broad anomaly of the susceptibility peaking at $T_{\mathrm{N}}$. So this anomaly is related to the progressive extension of the "fluctuating" phase throughout the sample as temperature increases; its maximum occurs when all the sample is in the paramagnetic phase. As to the thermal variation of the hyperfine field, which abruptly drops from $8 \mathrm{~T}$ at $160 \mathrm{~K}$ to zero at $170 \mathrm{~K}$, it strongly suggests that the transition has a pronounced first order character. The coexistence of ordered and fluctuating/paramagnetic states is also a typical feature of a first order transition. However, it usually occurs over a much smaller temperature interval than observed in MnGe.

The hyperfine field at $4.2 \mathrm{~K}$ is unusually small (16.1 T) compared to that in metallic $\mathrm{Fe}(34 \mathrm{~T})$ or in insulating oxides $(50 \mathrm{~T})$ at the same temperature. This rather low value suggests that the hyperfine field is a transferred field, i.e., it is due to the spin polarization of the $\mathrm{Mn}$ conduction bands at the $\mathrm{Fe}$ site. Since the latter is proportional to the Mn moment, the hyperfine field should show the same behavior. When fitting the spectra, in a first step, to a standard six-line pattern with Lorentzian-shaped lines, one observes the following: (i) some small misfits in the wings, hinting at the presence of a narrow non-Lorentzian distribution of hyperfine fields; (ii) a misfit at the left-hand inner line (marked by green arrows in Fig. 3): this line is too intense when compared to the right-hand inner line; (iii) a vanishingly small effective quadrupolar parameter which, in first analysis, can be due either to an actually zero electric field gradient (EFG) at the nucleus [although the noncubic Fe (Mn) site symmetry allows for a nonzero EFG] or to an effective zero EFG, due for instance to the fact that the hyperfine field is directed at the "magic angle" $\theta_{m}=a \cos (1 / \sqrt{3})=54.7^{\circ}$ with respect to the principal axis of the EFG at the Mn site, which is the [111] axis.

In a second fitting step, the helical nature of the magnetic ordering in MnGe must be taken into account. The neutron diffraction data [15] show that the Mn moments lie in the (001) plane according to an incommensurate helix (becoming commensurate below $40 \mathrm{~K}$ ) with propagation vector along [001]. Assuming that the Fe moment "follows" the Mn moment, the former is then statistically homogeneously distributed according to a helical order in the (001) plane. We have computed the corresponding spectrum by diagonalizing the full hyperfine Hamiltonian, with the EFG axis along [111] and the hyperfine field homogeneously distributed in the (001) plane. In case the EFG in the ordered phase is zero, like in the paramagnetic phase, then the spectrum is a regular broadened six-line pattern and the asymmetry of the two inner lines cannot be reproduced. In order to obtain such an asymmetry, one must assume the presence of a nonzero EFG; then the angle between the EFG axis [111] and the hyperfine field in the (001) plane comes into play in defining the hyperfine energies. Due to the helicoidal arrangement, this angle varies, giving rise to a small distribution of hyperfine energies resulting in an asymmetry of the two inner lines of the spectrum. Furthermore, in order to account for the non-Lorentzian shape of the hyperfine field distribution, it is convenient to fit the spectrum with a histogram of hyperfine fields. The corresponding fit, together with the obtained histogram, is represented in Fig. 5. The correct asymmetry of the inner lines was obtained with a small negative quadrupolar parameter $\Delta E_{Q}=-0.38 \mathrm{~mm} / \mathrm{s}$, with $\Delta E_{Q}=\frac{e Q V_{Z Z}}{2}$, where $Q$ is the electric quadrupole moment of the excited nuclear state and $V_{Z Z}$ is the component of the $\mathrm{EFG}$ along $\mathrm{OZ}=[111]$. The fit shown in Fig. 5 represents the experimental data satisfactorily; in particular it yields a vanishing mean spectral EFG. The obtained narrow hyperfine field distribution probably arises from small inhomogeneities in the sample.

It must be noted that the presence of a nonzero EFG in the magnetic phase, whereas the EFG is zero in the paramagnetic phase, can only occur if a distortion takes place in the magnetically ordered phase, in agreement with the neutron diffraction findings [15].

\section{NEUTRON DIFFRACTION}

\section{A. Ambient pressure results}

Neutron diffraction at ambient pressure was carried out on the diffractometer G4.1 (LLB), in similar conditions as in Ref. [24], but with increased statistics. To study the fluctuating chiral phase, many patterns were taken in the transition region. We focus here on the low angle region. Ferromagnetic correlations are observed well above $T_{\mathrm{N}}$ up to about $250 \mathrm{~K}$, and their intensity is maximum at $T_{\mathrm{N}}$ (Fig. 6, upper panel), where a magnetic satellite of the $q=0$ Bragg peak appears (Fig. 6, middle panel). When temperature decreases below $T_{\mathrm{N}}$ the satellite moves towards higher angles and its intensity increases. Ferromagnetic correlations persist below $T_{\mathrm{N}}$ down to about $100 \mathrm{~K}$, as shown by the presence of an angular dependent background below the satellite. 


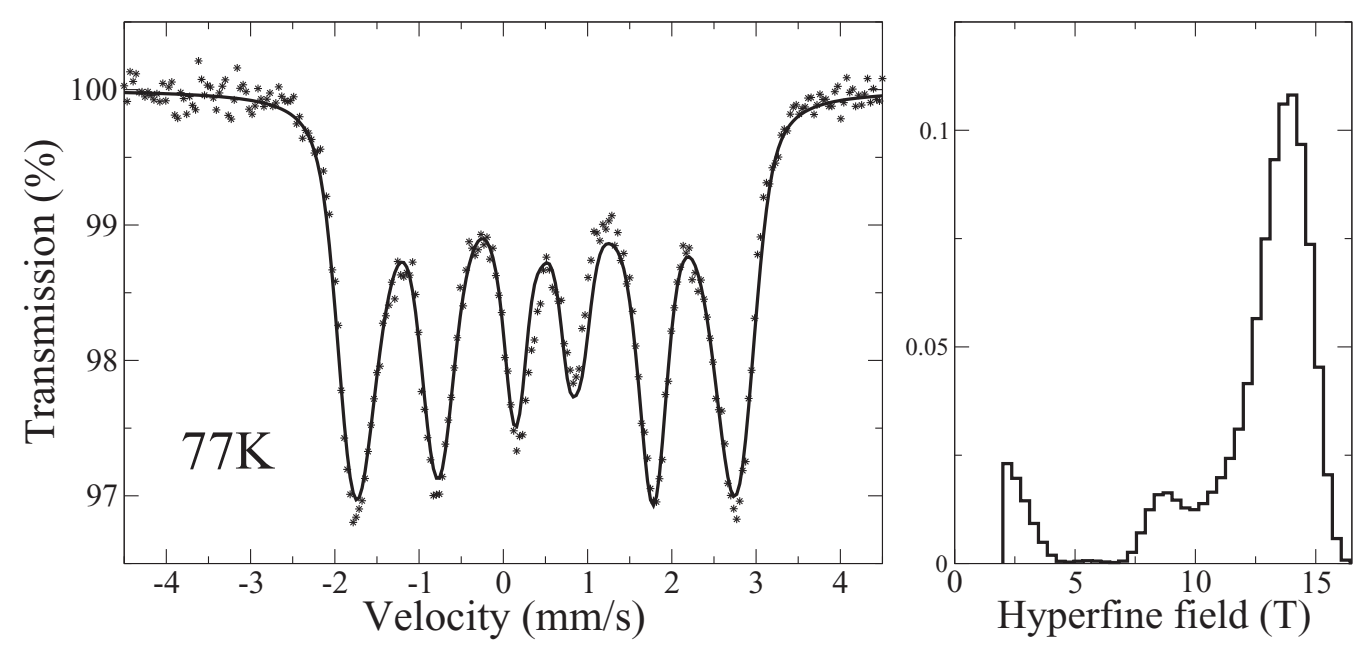

FIG. 5. ${ }^{57} \mathrm{Fe}$ Mössbauer spectrum (right panel) and hyperfine field distribution (right panel) at $77 \mathrm{~K}$ in Fe-doped MnGe sample 1. The line is a fit to a line shape taking into account the spiral magnetic structure of the Mn ions and a nonvanishing EFG at the Fe site (see text).

The intensity and linewidth of these correlations show a critical behavior, with a dynamical narrowing and maximum of intensity at $T_{\mathrm{N}}$ (see Fig. 6, lower panel). Unexpectedly, the ferromagnetic correlations are present in a very large temperature range $\left(T_{N} \pm 70 \mathrm{~K}\right)$, which corresponds to the region of spin fluctuations identified from Mössbauer spectroscopy and susceptibility. Good fits of the correlation peaks were obtained with a Gaussian line shape centered at $2 \theta=0^{\circ}$, allowing one to follow the temperature dependence of the intensity and to extract a typical length scale $D(T)$. It is identified as the domain size, using Scherrer's formula [25]:

$$
D=2 \sqrt{\frac{\ln 2}{\pi}} \frac{\lambda}{\Delta(2 \theta)_{G} \cos \theta},
$$

where $\Delta(2 \theta)_{G}$ is the full width at half maximum (FWHM) of the Gaussian line, of the correlations probed in the experimental $2 \theta$ window (Fig. 6). The small signal measured at $10 \mathrm{~K}$ and $330 \mathrm{~K}$, namely well below or well above $T_{\mathrm{N}}$, is attributed to nuclear diffraction of the powder grains. Subtracting this signal from the data allows the purely magnetic contribution to be extracted, yielding a slight increase of the domain size without changing its temperature dependence. However, this magnetic contribution cannot be fitted to a Lorentzian line shape, by contrast to what usually holds for critical phenomena.

Below $T_{\mathrm{N}}$, the ferromagnetic contribution from the fluctuating Mn moments coexists with an intense satellite of the $q=0$ Bragg peak (hereafter referred to as "zero satellite"), arising from the moments involved in the static helical order. The average ordered moment $m_{\mathrm{av}}(T)$ is deduced from FULLPROF refinement (Fig. 7, upper panel), assuming the same magnetic and crystal structure as in Ref. [15]. Its value at low temperature $\left(m=1.80 \mu_{\mathrm{B}}\right.$ at $\left.2 \mathrm{~K}\right)$ agrees rather well with the moment value deduced from the high field magnetization data in the almost fully polarized ferromagnetic state $\left(m=1.65 \mu_{\mathrm{B}}\right.$ at $2 \mathrm{~K}$ and $14 \mathrm{~T}$ ). Its temperature dependence is plotted in the lower panel of Fig. 7 together with that of the scaled hyperfine field. The two quantities are proportional at low temperature up to $100 \mathrm{~K}$, but they start to deviate in the temperature range $100-170 \mathrm{~K}$ where the fluctuating chiral phase is stabilized. In this region, the magnetic moment deduced from neutron diffraction decreases with heating more steeply that the hyperfine field. We attribute this deviation to the presence of the fraction $F(T)$ of rapidly fluctuating correlated spins evaluated from the Mössbauer spectra (see Fig. 3). The moment $m_{\text {hel }}(T)$ associated with the helical order alone is then evaluated as $m_{\text {hel }}(T)=\frac{m_{\text {av }}(T)}{\sqrt{1-F(T)}}$. As shown in Fig. 7 (lower panel), this quantity scales very well with the hyperfine field in the whole temperature range up to $T_{\mathrm{N}}$, which supports our previous assumption of a transferred hyperfine field at the Fe site.

\section{B. Stress-induced magnetic textures}

Neutron experiments under nonhydrostatic pressure were carried out at the Laboratoire Léon Brillouin on the G6.1 diffractometer (incident neutron wavelength $4.745 \AA$ ), using focusing devices and a new 2D multidetector, made of 16 horizontal tubular detectors. The sample was inserted in a Kurchatov-LLB sapphire anvil cell [26], with $\mathrm{NaCl}$ as transmitting medium. The pressure was calibrated by ruby fluorescence. With this setup, the uniaxial pressure component or stress can be estimated to 0.3(1) GPa [26,27]. The stress component is oriented along the principal axis of the pressure cell, which corresponds to the $Z$ axis of the detector (Fig. 8). We measured patterns at four pressures between 0.8 and $3.2 \mathrm{GPa}$. The cell was inserted in a helium cryostat and, for each pressure, we measured at several temperatures down to $5 \mathrm{~K}$. Measurements at ambient pressure were also performed with the same multidetector for comparison.

Below $T_{\mathrm{N}}$, the zero satellite appears as an intensity ring on the 2D multidetector [Fig. 8(e)]. We notice that its intensity seems to be reduced in the vertical direction due to the anisotropic pixel shape of the multitube detector, with limited resolution along the $Z$ axis (Fig. 8). This instrumental anisotropy is taken into account in the simulation by applying a filter to an incident isotropic signal.

Under pressure the distribution of intensity over the satellite ring changes dramatically [Fig. 8(f)]. Even at the smallest pressure of $0.8 \mathrm{GPa}$, the intensity is strongly enhanced along 

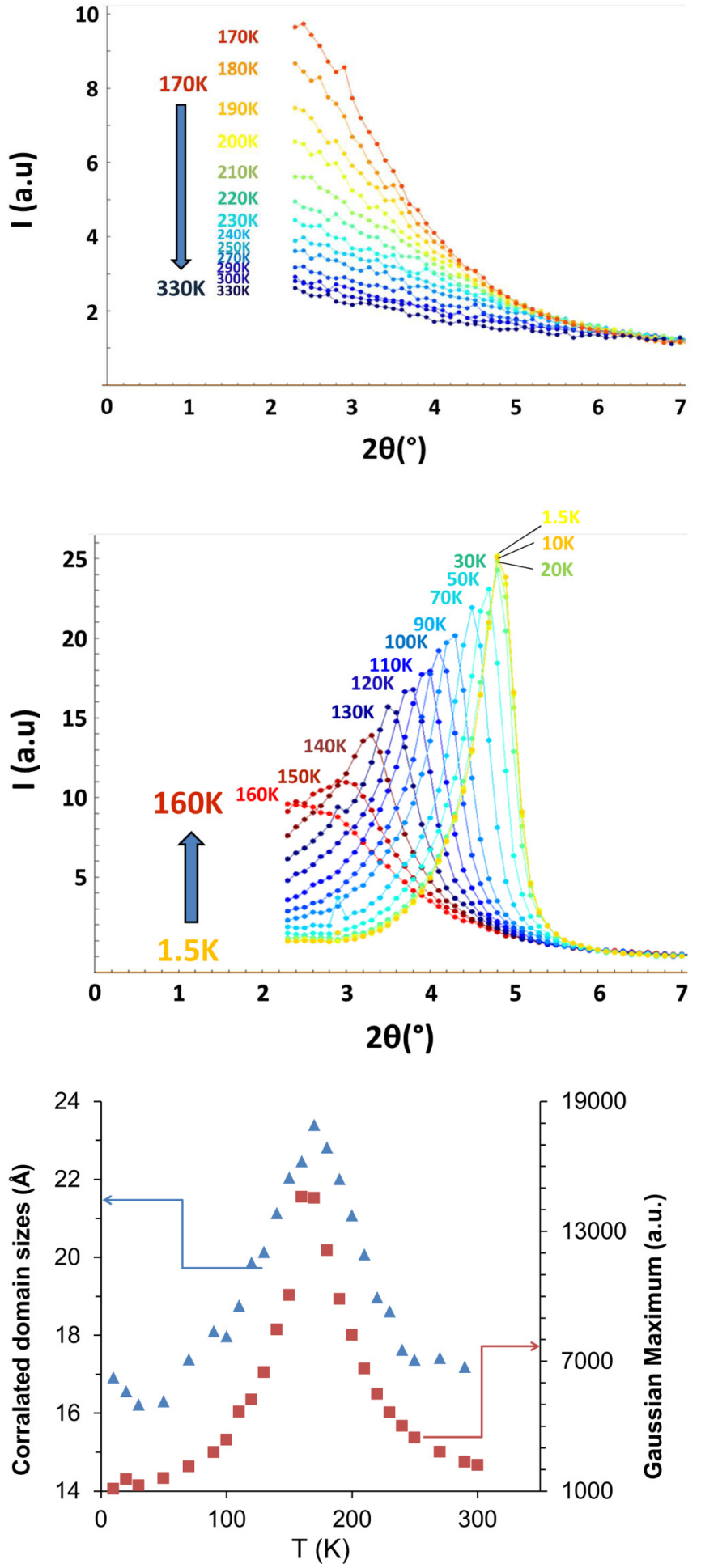

FIG. 6. (Color online) Upper panel: diffraction pattern at low angle vs temperature above $T_{\mathrm{N}}$. Middle panel: diffraction pattern at low angle vs temperature below $T_{\mathrm{N}}$ : the zero satellite is superimposed on a low angle signal fitted with a Gaussian line shape centered at $2 \theta=0^{\circ}$. Lower panel: temperature dependence of the maximum of the Gaussian line used to fit the low angle signal and of the correlated domain size calculated by Scherrer's formula [25].

the $Z$ axis. This corresponds to a magnetic texture effect, which practically does not depend on the value of the applied pressure. It is induced by the uniaxial component of the applied
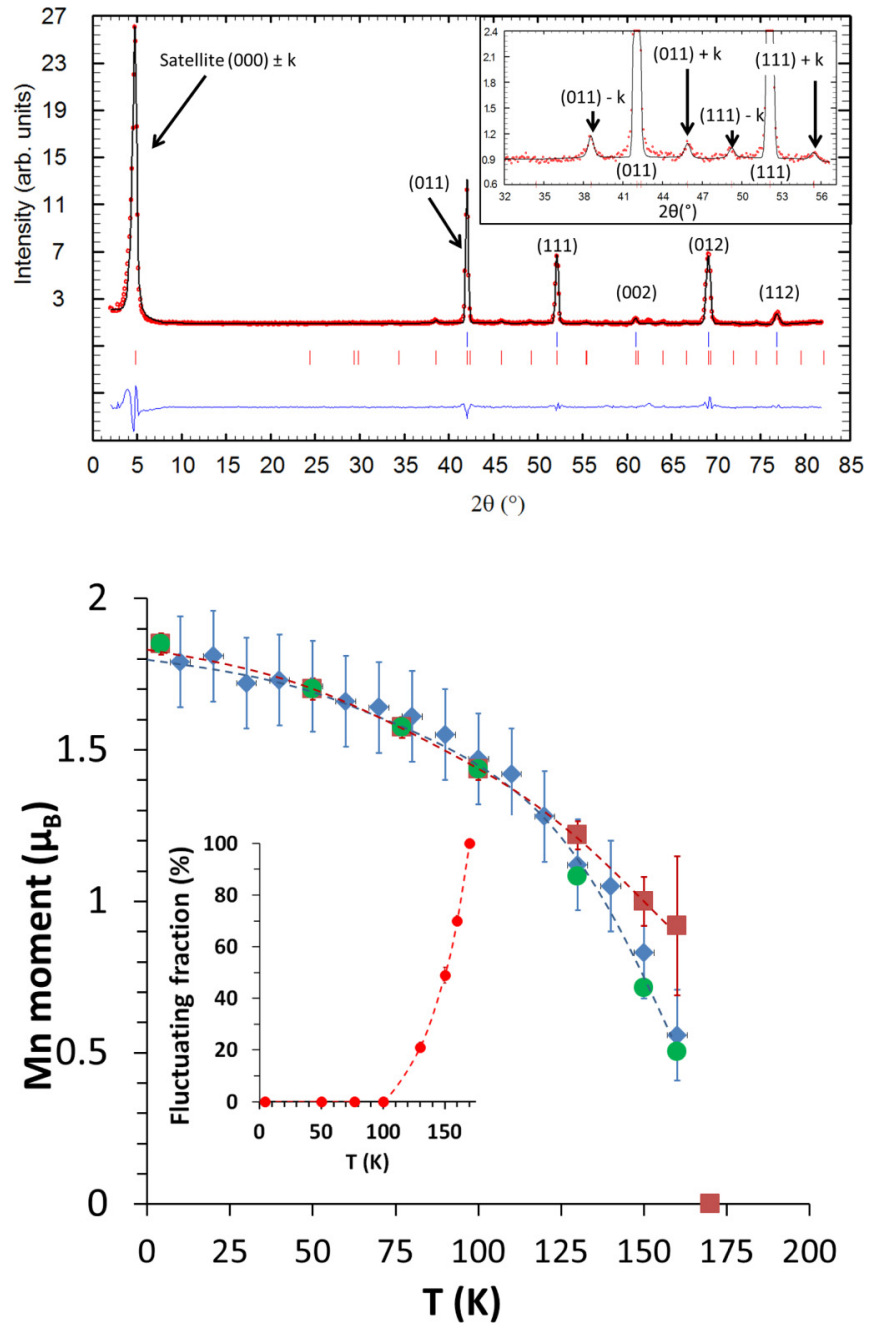

FIG. 7. (Color online) Upper panel: experimental powder diffraction pattern of $\mathrm{MnGe}$ at $1.5 \mathrm{~K}$ (red dots) and FULLPROF refinement (black line). The difference pattern is the blue line; upper (lower) tick marks show the positions of the nuclear (magnetic) Bragg peaks. Inset: zoom around the (011) and (111) Bragg peaks showing the small magnetic satellites at high angles. Lower panel: comparison of the temperature dependence of the average $\mathrm{Mn}$ ordered magnetic moment obtained by neutron diffraction (blue diamonds) and by Mössbauer measurements in sample 1 (red squares). Green dots show the scaled Mössbauer moments taking into account the paramagnetic fraction in order to compare to neutron data. Lines are guides for eyes. Inset: fluctuating fraction vs temperature for the Mössbauer sample 1.

pressure, evaluated to $0.3(1) \mathrm{GPa}$ and independent of pressure within our experimental accuracy.

This magnetic texture can be readily associated with a preferential orientation of the helical axes along the $Z$ axis of the pressure cell. The anisotropic distribution of intensity in the detector plane (Fig. 9) can be accounted for by a Gaussian distribution of the helical axes around the $Z$ axis, in the simple assumption of a multidomain state.

The magnetic cross section is written as

$$
\frac{d \sigma_{M}}{d \Omega}(\mathbf{Q})=N \frac{(2 \pi)^{3}}{v_{0}} \sum_{\mathbf{k}} \sum_{\boldsymbol{\tau}}\left|\mathbf{F}_{M \perp}(\mathbf{Q})\right|^{2} \delta(\mathbf{Q}-\mathbf{k}-\boldsymbol{\tau}),
$$


Ambient Pressure

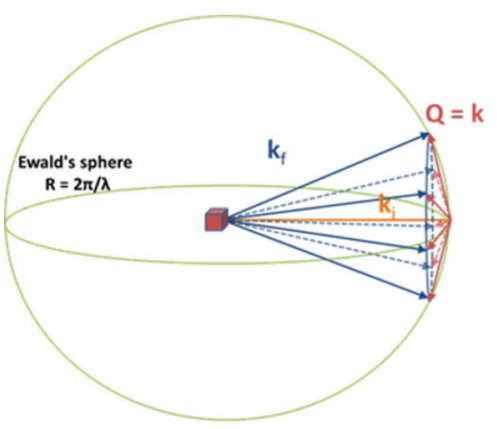

With Uniaxial Pressure $~ 0.3 \mathrm{GPa}$

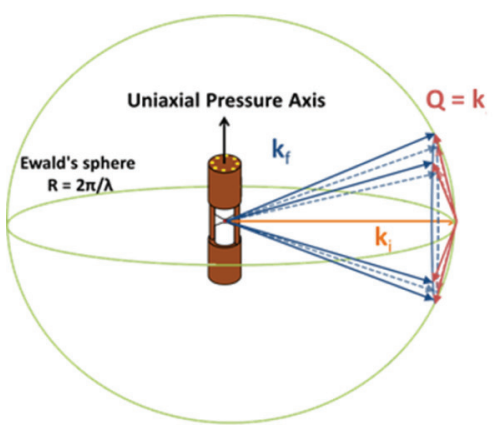

(a)

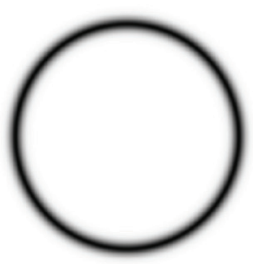

(b)

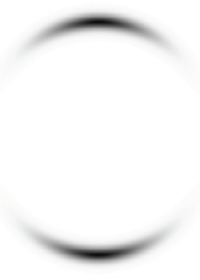

(c)

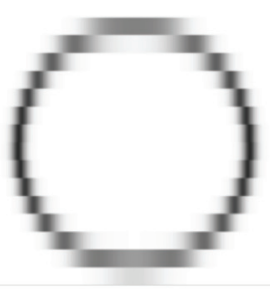

(d)

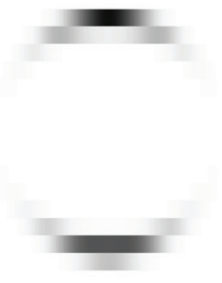

(e)

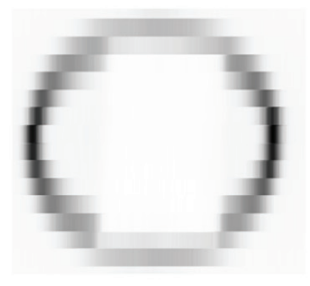

(f)

FIG. 8. (Color online) Illustration of a diffraction process for a powder without texture (upper panel) and with texture (lower panel) in MnGe. Upper panel: magnetic zero satellite without texture (isotropic distribution): (a) simulated on an isotropic 2D detector; (c) simulated on a multitubes detector as installed on the G6.1 diffractometer (LLB); (e) measured on G6.1 at $5 \mathrm{~K}$ and ambient pressure. Lower panel: magnetic zero satellite with texture oriented along the vertical direction with a Gaussian distribution of orientations (ODF): (b) simulated on an isotropic 2D detector; (d) simulated on a multitubes detector as installed on G6.1; (f) measured on G6.1 at $5 \mathrm{~K}$ and $3.2 \mathrm{GPa}$. Under pressure the intensity is enhanced along the axis of the pressure cell, due to the reorientation of helical domains.

where $\boldsymbol{\tau}$ are reciprocal lattice vectors, $\mathbf{k}$ are the wave vectors associated with the Fourier component of the magnetic distribution, and $F_{M}$ is the magnetic structure factor defined

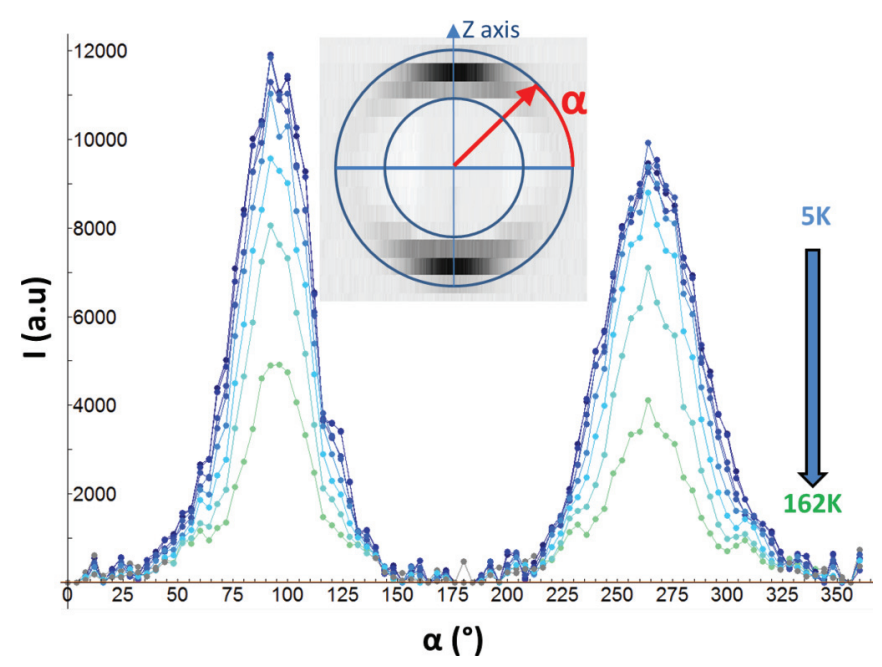

FIG. 9. (Color online) Diffracted intensity of the magnetic zero satellite vs the orientation angle $\alpha$ in the detector plane. The satellite is measured at $P=3.2 \mathrm{GPa}$ at different temperatures. as

$$
\begin{gathered}
\mathbf{F}_{M \perp}=\mathbf{F}_{M}-\left[\mathbf{F}_{M} \cdot \widehat{\mathbf{Q}}\right] \widehat{\mathbf{Q}}, \\
\mathbf{F}_{M}=\frac{\gamma e^{2}}{2 m_{e} c^{2}} \sum_{i, j} \mathbf{m}_{i, j} f(\mathbf{Q}) e^{i \mathbf{Q} \cdot \mathbf{R}_{i, j}} \cdot e^{-W_{i, j},}
\end{gathered}
$$

with $f(\mathbf{Q})$ the magnetic form factor, and $\mathbf{m}_{i, j}$ and $\mathbf{R}_{i, j}$ the magnetic moment and position of atom $j$ of lattice unit $i$. $W_{i, j}$ is the Debye-Waller factor.

For single helix the moment of atom $j$ of lattice unit $i$ can be written as

$$
\mathbf{m}_{i, j}=\mathbf{m}_{0}\left[\cos \left(\mathbf{k}_{h} \cdot \mathbf{R}_{i, j}\right) \mathbf{e}_{1}+\sin \left(\mathbf{k}_{h} \cdot \mathbf{R}_{i, j}\right) \mathbf{e}_{2}\right],
$$

where $\mathbf{e}_{1}$ and $\mathbf{e}_{2}$ are two orthogonal unit vectors perpendicular to the helix axis $\mathbf{k}_{h}$.

Equations (5) and (2) show that helical spin structures yield satellites of the nuclear peaks ( $\boldsymbol{\tau}$ ) located at $\mathbf{Q}=\boldsymbol{\tau} \pm \mathbf{k}_{h}$. At ambient pressure, the random orientation of the helical axes in the randomly oriented powder grains yields an isotropic distribution of intensity in the detector plane. The magnetic texture induced under stress is readily linked with a preferential orientation of the helical axes. As a single domain with helical axis along $Z$ yields only two satellites $\left( \pm \mathbf{k}_{h}\right)$ in the detector plane, the distribution of intensity measured in Fig. 9, which obeys a Gaussian line shape with a maximum of intensity along $Z$, is then readily associated with a Gaussian distribution of 
orientations of the helical axes along the $Z$ axis. This magnetic texture persists in the whole pressure-temperature range up to $T_{\mathrm{N}}$, and the Gaussian width $\left[\mathrm{FWHM} \simeq 45(5)^{\circ}\right]$ is independent of pressure and temperature. With the assumption of a strong magnetic texture, where all helical axes contribute in the same way to the scattering in the detector plane, this means that $95 \%$ of them belong to a cone of vertical axis and $76^{\circ}$ solid angle and $98 \%$ are situated in a similar cone of $90^{\circ}$ aperture.

\section{DISCUSSION}

\section{A. Magnetic ground state}

In bulk MnGe powder below $T_{\mathrm{N}}$, applying a stress induces a uniaxial magnetic anisotropy. This reveals a magnetoelastic coupling, also observed for instance in thin films of MnSi under epitaxial strains $[28,29]$. Here we find that the anisotropy axis is a hard axis, so that the majority of the helical axes reorients along the stress axis. The resulting magnetic texture is very similar to that induced by a magnetic field in bulk MnGe powder [19]. However, whereas in Ref. [19] the field induced reorientation occurs through grain reorientation, in our heavily compressed sample, the isotropic pressure component is much greater than the uniaxial one $\left(\mathrm{P}_{\text {iso }} / \mathrm{P}_{u}\right) \sim 3-10$ and the grains cannot reorient.

Therefore, the most natural explanation for the stress induced magnetic texture is the growth of magnetic domains within the ensemble of randomly oriented grains. Recalling that, in $\mathrm{MnGe}$, the helical axes are oriented along $\langle 001\rangle$ crystal axes, each grain can select the [001] axis closest to the $Z$ axis as the preferential axis to minimize the magnetoelastic energy. This process occurs in powder samples when nonhydrostatic pressures are applied below a magnetostructural transition. It naturally explains the value of the Gaussian linewidth, yielding $98 \%$ helical axes within a cone of $90^{\circ}$ aperture, since whatever the orientation of a given grain, it will possess a [001] axis in such a cone.

We notice that when the effect of textures is averaged over the 2D detector, we obtain an excellent agreement between these data and those obtained under hydrostatic pressure, as concerns the pressure dependence of the Néel transition, propagation vector, and magnetic moment (see Ref. [17] and its Supplemental Material). This confirms our analysis and shows that there is no deep change in the magnetic structure in this pressure range.

As a natural consequence, our results suggest that the ambient pressure and zero field ground state is a multidomain state made of single- $k$ helical domains of random orientations, and not a lattice of skyrmions and antiskyrmions, namely a complex multi- $k$ structure where the selection of single- $k$ single-domain state under stress would be energetically less favorable. Similarly in single crystals, applying uniaxial stress is a usual way to distinguish single- $k$ from multi- $k$ magnetic structures [30].

Our simple description of the bulk MnGe ground state is supported by several observations and calculations in the B20 family. In bulk B20 samples allowing a direct observation of the SK lattice by single crystal neutron diffraction $[8,31,32]$, this lattice was observed only in the vicinity of $T_{\mathrm{N}}$ and under applied field, but not in the zero field ground state, so that $\mathrm{MnGe}$ would stand as an exception. Thermal fluctuations [8] and/or a reduced stiffness of the magnetization modulus [28,33] are needed to stabilize the SK phase in the bulk state, which explains its small extension with temperature. Taking into account the high values of the anisotropy and saturation fields of MnGe (7 T and $12 \mathrm{~T}$, respectively, as compared to $0.1 \mathrm{~T}$ and $0.6 \mathrm{~T}$ in $\mathrm{MnSi}$ ), if the involved planar and $\mathrm{DM}$ anisotropies were strong enough to favor a condensed skyrmionic phase down to $T=0 \mathrm{~K}$, this phase should exist and even be favored by large fields in contrast with experimental observation using neutrons [19].

On the other hand, in MnGe at low temperature, the evolution of the magnetization and small angle neutron scattering with the magnetic field can be easily explained assuming a multidomain ground state in zero field, rather than the SK lattice previously proposed [19]. The field irreversibilities can naturally be related to the persistence of small helical domains blocked along a [001] axis at a large angle with the field axis. If the grains align along the magnetic field, such domains would be oriented at $90^{\circ}$ of the field and yield a neutron intensity in the direction $Q \perp H$, as experimentally observed [19].

Then what could be the origin of the giant THE of MnGe? Although helical or conical structures contribute to the magnetoresistance, they do not yield THE since the scattered electrons do not carry a Berry phase. The fluctuating chiral phase could play a role just below $T_{\mathrm{N}}$, but the strong temperature independent value of the transverse Hall resistivity at very low temperature remains to be explained. Other contributions to the THE related to a multicarrier effect [34] could perhaps be at play, as recently proposed for $\mathrm{Mn}_{1-x} \mathrm{Fe}_{x} \mathrm{Si}$ thin films [35]. A THE has also been reported in fanlike noncoplanar helimagnet $\mathrm{MnP}$ [36], where the spin structure has no net skyrmion number.

\section{B. Transition region}

We have evidenced a specific feature of MnGe, namely a broad transition temperature range extending in our sample up to $70 \mathrm{~K}$ on each side of $T_{\mathrm{N}}$, and we have determined its characteristics by combining magnetization, Mössbauer, and neutron data. This transition range is magnetically inhomogeneous, but the different magnetic regions belong to the same crystal phase. It presents some analogies with the non-Fermi liquid phase identified in $\mathrm{MnSi}$ and $\mathrm{FeGe}$, but in $\mathrm{MnGe}$ it extends in a much larger temperature range above $T_{\mathrm{N}}$ and persists well below in the ordered state. Below $T_{\mathrm{N}}$, ferromagnetically correlated rapidly fluctuating spins coexist with frozen moments involved in the helices. We have measured the relative fraction of the Mn moments involved in each type of state thanks to a quantitative comparison between neutron and Mössbauer data. We notice that the correlation length of the fluctuating moments (10-30 in our experimental range) and that of the helices (limited by the instrumental resolution, namely greater than $500 \AA$ ) differ by at least an order of magnitude, suggesting that the system segregates into different subphases. Above $T_{\mathrm{N}}$, the diffraction signal from the long range ordered helices vanishes and the Mössbauer spectrum consists of a single line. Susceptibility and neutron data show that spin correlations persist up to about $240 \mathrm{~K}$, and field cooling irreversibilities extend even above. It suggests that above $T_{\mathrm{N}}$ chiral fluctuations such as short range 
ordered helices $[37,38]$ still coexist with a few large scale quasistatic inhomogeneities.

This crossover region is also observed in the data obtained in Refs. [14,19]. In Fig. 1(a) of Ref. [14], a broad peak in the magnetic susceptibility is present, but it is rather asymmetric around a maximum at $T_{N} \simeq 180 \mathrm{~K}$ : it increases sharply above $150 \mathrm{~K}$ and decreases smoothly above $T_{\mathrm{N}}$, following the behavior of the ferromagnetic correlation contribution seen on Fig. 2(g) of Ref. [19] which decreases smoothly above $T_{\mathrm{N}}$ but abruptly disappears at $142 \mathrm{~K}$. This means that this crossover region is an intrinsic feature of $\mathrm{MnGe}$ but its temperature expansion and shape are sample dependent and can be probed by susceptibility measurements.

Mössbauer experiments have shown that the transition is first order in MnGe. In chiral magnets of the B20 structure, the onset of helical order through a first order transition is expected from theory [39]. The DM interaction can be expressed as a socalled Lifschitz invariant in the Landau free energy $[33,40]$ so that no second order transition is expected. Going beyond the Bak-Jensen model, in MnSi the features of the transition just above $T_{\mathrm{N}}$ have been analyzed as an example of the Brazovskii mechanism $[41,42]$. But the large crossover region around $T_{\mathrm{N}}$ and the persistence of spin fluctuations well below $T_{\mathrm{N}}$ is specific to $\mathrm{MnGe}$. Another specificity of $\mathrm{MnGe}$ is the onset at $T_{\mathrm{N}}$ of a lattice distortion, probed by neutron diffraction and Mössbauer spectroscopy, which has not been reported in other B20 magnets.

This crossover regime and the magnetostructural anomalies are possibly related to the peculiar band structure of $\mathrm{MnGe}$ which enables a high-spin (HS) to low-spin (LS) transition [18]. Such a transition could be in principle encompassed either with temperature or pressure [18]. Our recent measurements combined with refined calculations [17] suggest that it indeed occurs in the ground state under very high pressures, yielding a two step collapse of the helical order.

With varying temperature, the observation of a HS-LS transition is not obvious, and we cannot ascertain it presently. We however notice that an inhomogeneous magnetic state, a crossover regime at the transition, and the occurrence of a structural anomaly are in qualitative agreement with a HS-LS transition. HS and LS regions are expected to have very different specific volumes, so that thermal or quantum fluctuations are needed to accommodate the volume mismatch. This yields an intermediate fluctuating magnetoelastic state, in a process which recalls that of invar alloys $[43,44]$. We speculate that this intermediate state is realized through a dynamical phase separation above $T_{\mathrm{N}}$, and through a phase segregation between static and fluctuating regions below $T_{\mathrm{N}}$.

\section{ACKNOWLEDGMENTS}

We are deeply thankful to O. L. Makarova for her help in the high-pressure experiments. We thank U. K. Rößler, C. Lacroix, K. Pappas, S. V. Grigoriev, and S. M. Stishov for many useful discussions and comments. The post doc training of M.D. is funded by the ANR (DYMAGE) and the Triangle de la Physique. The stays of O. L. Makarova at LLB in 2012-2013 are funded by the Triangle de la Physique and Labex PALM. A public grant from the "Laboratoire d'Excellence Physics Atom Light Mater" (Labex PALM) was overseen by the French National Research Agency (ANR) as part of the "Investissements d'Avenir" program (Grant No. ANR-10-LABX-0039-PALM). A.V.T. acknowledges the support of the Russian Foundation for Basic Research (Grant No. 14-02-00001).
[1] C. Felser, Ang. Chem. Int. Ed. 52, 1631 (2013).

[2] A. Fert, V. Cros, and J. Sampaio, Nat. Nanotechnol. 8, 152 (2013).

[3] X. Z. Yu, Y. Onose, N. Kanazawa, J. H. Park, J. H. Han, Y. Matsui, N. Nagaosa, and Y. Tokura, Nature (London) 465 , 901 (2010)

[4] N. Romming, C. Hanneken, M. Menzel, J. E. Bickel, B. Wolter, K. von Bergmann, A. Kubetzka, and R. Wiesendanger, Science 341, 636 (2013).

[5] F. Jonietz, S. Muehlbauer, C. Pfleiderer, A. Neubauer, W. Muenzer, A. Bauer, T. Adams, R. Georgii, P. Boeni, R. A. Duine, K. Everschor, M. Garst, and A. Rosch, Science 330, 1648 (2010).

[6] A. Neubauer, C. Pfleiderer, B. Binz, A. Rosch, R. Ritz, P. G. Niklowitz, and P. Böni, Phys. Rev. Lett. 102, 186602 (2009).

[7] N. Nagaosa and Y. Tokura, Nat. Nanotechnol. 8, 899 (2013).

[8] S. Mühlbauer, B. Binz, F. Jonietz, C. Pfleiderer, A. Rosch, A. Neubauer, R. Georgii, and P. Böni, Science 323, 915 (2009).

[9] C. Pfleiderer, T. Adams, A. Bauer, W. Biberacher, B. Binz, F. Birkelbach, P. Böni, C. Franz, R. Georgii, M. Janoschek, F. Jonietz, T. Keller, R. Ritz, S. Mühlbauer, W. Münzer, A. Neubauer, B. Pedersen, and A. Rosch, J. Phys.: Condens. Matter 22, 164207 (2010).
[10] T. Adams, S. Mühlbauer, C. Pfleiderer, F. Jonietz, A. Bauer, A. Neubauer, R. Georgii, P. Böni, U. Keiderling, K. Everschor, M. Garst, and A. Rosch, Phys. Rev. Lett. 107, 217206 (2011).

[11] C. Pfleiderer, D. Reznik, L. Pintschovius, H. v. Lohneysen, M. Garst, and A. Rosch, Nature (London) 427, 227 (2004).

[12] C. Pappas, E. Lelièvre-Berna, P. Falus, P. M. Bentley, E. Moskvin, S. Grigoriev, P. Fouquet, and B. Farago, Phys. Rev. Lett. 102, 197202 (2009).

[13] A. Hamann, D. Lamago, T. Wolf, H. v. Löhneysen, and D. Reznik, Phys. Rev. Lett. 107, 037207 (2011).

[14] N. Kanazawa, Y. Onose, T. Arima, D. Okuyama, K. Ohoyama, S. Wakimoto, K. Kakurai, S. Ishiwata, and Y. Tokura, Phys. Rev. Lett. 106, 156603 (2011).

[15] O. L. Makarova, A. V. Tsvyashchenko, G. Andre, F. Porcher, L. N. Fomicheva, N. Rey, and I. Mirebeau, Phys. Rev. B 85, 205205 (2012).

[16] Y. Shiomi, N. Kanazawa, K. Shibata, Y. Onose, and Y. Tokura, Phys. Rev. B 88, 064409 (2013).

[17] M. Deutsch, O. L. Makarova, T. C. Hansen, M. T. FernandezDiaz, V. A. Sidorov, A. V. Tsvyashchenko, L. N. Fomicheva, F. Porcher, S. Petit, K. Koepernik, U. K. Rößler, and I. Mirebeau, Phys. Rev. B 89, 180407 (2014).

[18] U. K. Rößler, J. Phys.: Conf. Ser. 391, 012104 (2012). 
[19] N. Kanazawa, J. H. Kim, D. S. Inosov, J. S. White, N. Egetenmeyer, J. L. Gavilano, S. Ishiwata, Y. Onose, T. Arima, B. Keimer, and Y. Tokura, Phys. Rev. B 86, 134425 (2012).

[20] A. Tsvyashchenko, J. Less Common Met. 99, L9 (1984).

[21] A. Tsvyashchenko, V. A. Sidorov, L. N. Fomicheva, V. N. Krasnorussky, R. A. Sadykov, J. D. Thompson, K. Gofryk, F. Ronning, and V. Y. Ivanov, Solid State Phenom. 190, 225 (2012).

[22] C. Thessieu, C. Pfleiderer, A. N. Stepanov, and J. Flouquet, J. Phys.: Condens. Matter 9, 6677 (1997).

[23] K. Koyama, T. Goto, T. Kanomata, and R. Note, Phys. Rev. B 62, 986 (2000).

[24] O. L. Makarova, I. Mirebeau, S. E. Kichanov, J. RodriguezCarvajal, and A. Forget, Phys. Rev. B 84, 020408 (2011).

[25] A. Guinier, Théorie et technique de la radiocristallographie: par A. Guinier,... 2e édition (Dunod, Paris, 1956).

[26] I. N. Goncharenko, High Press. Res. 24, 193 (2004).

[27] I. Mirebeau, I. N. Goncharenko, G. Dhalenne, and A. Revcolevschi, Phys. Rev. Lett. 93, 187204 (2004).

[28] A. B. Butenko, A. A. Leonov, U. K. Rößler, and A. N. Bogdanov, Phys. Rev. B 82, 052403 (2010).

[29] M. N. Wilson, A. B. Butenko, A. N. Bogdanov, and T. L. Monchesky, Phys. Rev. B 89, 094411 (2014).

[30] J. Rossat-Mignod, G. Lander, and P. Burlet, Handbook on the Physics and Chemistry of the Actinides (North-Holland, Amsterdam, 1984), Chap. 6, pp. 416-513.

[31] T. Adams, A. Chacon, M. Wagner, A. Bauer, G. Brandl, B. Pedersen, H. Berger, P. Lemmens, and C. Pfleiderer, Phys. Rev. Lett. 108, 237204 (2012).
[32] E. Moskvin, S. Grigoriev, V. Dyadkin, H. Eckerlebe, M. Baenitz, M. Schmidt, and H. Wilhelm, Phys. Rev. Lett. 110, 077207 (2013).

[33] U. Rößler, A. Bogdanov, and C. Pfleiderer, Nature (London) 442, 797 (2006).

[34] J. Ziman, Principles of the Theory of Solids (Cambridge University Press, Cambridge, UK, 1972).

[35] T. Yokouchi, N. Kanazawa, A. Tsukazaki, Y. Kozuka, M. Kawasaki, M. Ichikawa, F. Kagawa, and Y. Tokura, Phys. Rev. B 89, 064416 (2014).

[36] Y. Shiomi, S. Iguchi, and Y. Tokura, Phys. Rev. B 86, 180404 (2012).

[37] S. V. Grigoriev, E. V. Moskvin, V. A. Dyadkin, D. Lamago, T. Wolf, H. Eckerlebe, and S. V. Maleyev, Phys. Rev. B 83, 224411 (2011).

[38] C. Pappas, E. Lelièvre-Berna, P. Bentley, P. Falus, P. Fouquet, and B. Farago, Phys. Rev. B 83, 224405 (2011).

[39] P. Bak and M. H. Jensen, J. Phys. C: Solid State Phys. 13, L881 (1980).

[40] Y. A. Izyumov, Sov. Phys. Usp. 27, 845 (1984).

[41] M. Janoschek, M. Garst, A. Bauer, P. Krautscheid, R. Georgii, P. Böni, and C. Pfleiderer, Phys. Rev. B 87, 134407 (2013).

[42] S. Brazovskii, Zh. Eksp. Teor Fiz. 68, 175 (1975) [Sov. Phys. JETP 41, 85 (1975)].

[43] T. Moriya and K. Usami, Solid State Commun. 34, 95 (1980).

[44] M. van Schilfgaarde, I. Abrikosov, and B. Johansson, Nature (London) 400, 46 (1999). 